\title{
Mid-infrared observations of GRS 1915+105 during plateau and flaring states
}

\author{
Y. Fuchs ${ }^{1}$, I. F. Mirabel ${ }^{1,2}$, and A. Claret $^{1}$ \\ 1 Service d'Astrophysique, CEA/Saclay, Orme des Merisiers Bât. 709, 91191 Gif-sur-Yvette, France \\ 2 Instituto de Astonomía y Física del Espacio / CONICET, cc67, suc 28. 1428 Buenos Aires, Argentina
}

Received 31 January 2003 / Accepted 21 March 2003

\begin{abstract}
We present mid-infrared (4-18 $\mu \mathrm{m})$ observations of the microquasar GRS 1915+105 obtained with ISOCAM, the camera on board the Infrared Space Observatory (ISO), in 1996 April and 1997 October. The first observation probably occurred during a flaring event with oscillating synchrotron emission. The 1997 observation occurred a few days before a major relativistic ejection, during a plateau state of inverted-spectrum radio emission and hard quasi-stable X-ray emission. The K-M giant donor star in GRS 1915+105 cannot account for the mid-IR emission and we discuss the possible additional components depending on two absorption laws. Thermal emission from dust seems unlikely. The flat mid-IR spectrum obtained during the plateau state is likely to be synchrotron emission. It would be the first evidence of the infrared extension of the radio synchrotron emission from the compact jets, although optically thin free-free emission from an X-ray driven-wind from the accretion disc cannot be excluded.
\end{abstract}

Key words. stars: individual: GRS 1915+105 - X-rays: binaries - infrared: stars - stars: circumstellar matter ISM: jets and outflows - stars: winds, outflows

\section{Introduction}

GRS $1915+105$ is a transient hard X-ray source discovered in 1992 with Granat/WATCH (Castro-Tirado et al. 1992, 1994). It is one of the brightest $\mathrm{X}$-ray source in the Galaxy, highly variable in intensity and activity (Harmon et al. 1992; Greiner et al. 1996). Intense flares are also observed in radio (Rodríguez \& Mirabel 1993) and led to the discovery of superluminal ejections with the VLA (Mirabel \& Rodríguez 1994) i.e. structures moving with an apparent velocity higher than the light speed on the sky-plane. This first superluminal movement observed in our Galaxy, very similar to the ones observed in quasars like 3C 279 (Cotton et al. 1979), confirmed the microquasar nature of GRS 1915+105. The microquasar term refers to Xray binaries showing radio (persistent or not) jets imitating at much smaller scales the radio lobes of quasars and radio galaxies (Mirabel et al. 1992; Mirabel \& Rodríguez 1999).

GRS 1915+105 has a radio (Mirabel et al. 1993b) and an IR (Mirabel et al. 1993a) counterpart, and is highly variable at all these wavelengths (Mirabel et al. 1994). Because of the high interstellar extinction along the line of sight to GRS 1915+105, the type of the companion star and thus the masses of the binary components were difficult to identify. According to Greiner et al. (2001b) the donor star is of K-M III type corresponding to a late-type low mass $\left(\sim 1-1.5 M_{\odot}\right)$ giant star.

Send offprint requests to: Y. Fuchs,

e-mail: yfuchs@discovery.saclay.cea.fr
Greiner et al. (2001a) found the following binary parameters: orbital period $P_{\text {orb }}=33.5$ days, mass function $f(M)=9.5 \pm$ 3.0 $M_{\odot}$ which is the lower limit to the mass of the compact object, using $M_{\mathrm{d}}=1.2 \pm 0.2 M_{\odot}$ for the mass of the donor star and $\theta=70^{\circ} \pm 2^{\circ}$ for the inclination angle implies $M_{\mathrm{c}}=14 \pm 4 M_{\odot}$ for the mass of the compact object which is the most massive stellar black hole candidate.

\subsection{Multi-wavelength variability}

GRS $1915+105$ is unique for its X-ray variability at all time scales from less than one second to several days (Greiner et al. 1996). RXTE observations of the source were classified in different classes (12 classes found by Belloni et al. 2000a and a new class discovered by Klein-Wolt et al. 2002) according to their flux and spectral properties and corresponding to transitions between 3 basic states. Flaring events seen in radio, nearIR ( $K$-band) and $X$-ray with various periodicity and intensity were classified in 3 classes by Eikenberry et al. (2000) as in the following.

The first class is composed of the giant flares that were observed in radio and X-ray (Mirabel \& Rodríguez 1994; Rodríguez \& Mirabel 1999; Fender et al. 1999a; Dhawan et al. 2000) and correspond to major ejection events followed as superluminal motions in radio images. These flares are characterized by very rapid rise time (less than 1 day) with an increase in the radio flux density by 1 or 2 orders of magnitude (reaching 
600-1000 mJy) and optically thin exponential decay lasting ten or so days (Foster et al. 1996). Continuous short period radio oscillations (between optically thin and thick states) can happen during the flux decrease (Fender et al. 1999a, 2002).

These 20 to 40 min quasi-periodic oscillations form the second type of flares, first observed in radio with the Ryle Telescope at $15 \mathrm{GHz}$ (Pooley 1995, 1996) and with the Very Large Array (VLA) at $3.6 \mathrm{~cm}$ (Rodríguez \& Mirabel 1997). These oscillations are very common as shown by the Ryle Telescope monitoring of Pooley \& Fender (1997) who associated them with soft X-ray dips on time-scale of $\sim 30 \mathrm{~min}$. The latter were discovered by Greiner et al. (1996) with RXTE PCA observations and were interpreted by Belloni et al. (1997a,b) as the repeated disappearance and refilling of the inner accretion disc. Near-IR oscillations were also observed by Fender et al. (1997) who suggested that they were the high frequency tail of radio synchrotron emission. Simultaneous near-IR and X-ray observations by Eikenberry et al. (1998a) revealed the correspondence between these X-ray dips/flares and near-IR ( $2.2 \mu \mathrm{m} K$-band) flares consistent with synchrotron emission from ejected plasma bubbles or "baby jets" undergoing adiabatic expansion losses. The synchrotron nature of these oscillations was confirmed by simultaneous near-IR/radio (Fender \& Pooley 1998) and near-IR/radio/X-ray (Mirabel et al. 1998) observations of these $\sim 30 \mathrm{~min}$ dip/flare cycles, with wavelengthdependent time delays (radio-radio or radio-infrared). Recently Fender et al. (2002) demonstrated that this time delay is variable between epochs, with a possible correlation with the oscillation amplitude, but they still cannot distinguish between models of discrete ejections (as the van der Laan 1966 model used by Mirabel et al. 1998) or shocks propagating along quasi-continuous flows (Blandford \& Konigl 1979; Kaiser et al. 2000).

The amplitudes of the quasi-periodic oscillations are about $40-50 \mathrm{mJy}$ in radio, or $\sim 70-80 \mathrm{mJy}$ for the largest and $\sim 300 \mathrm{mJy}$ in millimetre (Fender \& Pooley 2000). In $K$-band the observed (not dereddened) amplitudes are $~ 2-3 \mathrm{mJy}$ (Fender et al. 1997; Mirabel et al. 1998; Fender \& Pooley 1998) $\sim 10 \mathrm{mJy}$ (Eikenberry et al. 1998a,b) and $\sim 20 \mathrm{mJy}$ (Fender \& Pooley 2000). Finally, Eikenberry et al. (2000) presented a third type of flare: faint (submillijansky) IR ( $K$-band) flares whose association with X-ray soft-dip/soft-flare behaviour is uncertain.

Here we present the first mid-IR ( 4 to $18 \mu \mathrm{m}$ ) observations of GRS $1915+105$ thanks to the sensitivity of ISOCAM, the IR camera on board the Infrared Space Observatory (ISO). These observations were part of a general campaign studying the interaction of the high energy emission from X-ray binaries with the surrounding material. In particular, thermal emission from heated dust was expected as the presence of dust arround GRS 1915+105 had been suggested by Mirabel et al. (1996) after observing a reddening at the time of a near-IR flare, and also by Martí et al. (2000) to explain the P Cygni profile of a He I line. In this paper we will first describe our ISOCAM observations and the difficulties in dereddening the data inherent to the mid-IR wavelength range. In Sects. 4 and 5 we show and interpret the results corresponding to two particular epochs in 1996 and 1997, when the source was in very different states: a possible flaring state in 1996 and the plateau state preceeding a giant outburst with superluminal ejections in 1997 October. We also discuss the nature of this mid-IR emission. Section 6 summarizes our conclusions.

\section{Observations}

\subsection{ISOCAM}

Mid-IR observations of GRS 1915+105 were carried out with the ISOCAM camera (Cesarsky et al. 1996) on board the Infrared Space Observatory (ISO) satellite mission as part of the Guaranteed and Open Time programmes. Table 1 shows the log of the observations for the two epochs in 1996 April and 1997 October.

\subsubsection{Data reduction}

The ISOCAM data were reduced with the Cam Interactive Analysis software (CIA, Delanay \& ISOCAM Interactive Analysis Team 2000) version 4.0, following the standard processing outlined in Starck et al. (1999). First a dark correction was applied, then a de-glitching to remove cosmic ray hits, followed by a transient correction to take into account memory effects. Pixels showing remnants of these effects were masked as well as side pixels insufficiently lit. The jitter correction was applied for $1.5^{\prime \prime} \times 1.5^{\prime \prime}$ resolution images. The flat-field correction used the automatic evaluation except for the $L W 1$ filter where the calibration flat field was used. Then individual images were combined into the final raster map and finally the pixel values were converted into milli-Jansky flux densities. No colour correction was applied.

\subsubsection{Images and photometry}

GRS 1915+105 appears on the ISOCAM images as a very faint point source, at the limit of the ISOCAM detection for a few filters in 1996. No extended emission was found, but the 0.3-0.6" long extensions seen by Sams et al. (1996b) at $2.2 \mu \mathrm{m}$ in 1995 July are far too small for ISOCAM spatial resolution. Note that these near-IR "jets" were suggested to be of synchrotron nature and variable, but their presence was never confirmed. Sams et al. (1996a) noted the disappearence of the jet since their previous observation and Eikenberry \& Fazio (1997) found no evidence of near-IR extended emission.

Achieving the photometry of GRS $1915+105$ was a delicate task as it is located close to a very bright mid-IR source (J2000 coordinates $\alpha=19^{\mathrm{h}} 15^{\mathrm{m}} 11.27^{\mathrm{s}}$ and $\left.\delta=10^{\circ} 56^{\prime} 27^{\prime \prime}\right)$. This source is the star visible in Fig. 2 of Mirabel et al. (1994) at the South-West of the ROSAT error circle on this $R$-band image, and in Fender et al. (1997) Fig. 1 (next to star "B") where it appears as already quite bright in the $K$-band. The $\sim 18^{\prime \prime}$ distance (center to center) between this star and GRS $1915+105$ corresponds to only 12, 6 and 3 pixels in our ISOCAM images depending on the spatial resolution. This star is very bright in all the ISOCAM images and its point spread function certainly overlap the one of GRS 1915+105. Thus we measured the integrated flux inside a tight circle around the source peak and we 
Table 1. Log of the IR observations of GRS $1915+105$.

\begin{tabular}{|c|c|c|c|c|c|c|}
\hline Date & $\mathrm{MJD}^{a}$ & $\begin{array}{l}\text { Timing } \\
\text { (UT) }\end{array}$ & Filter & $\begin{array}{c}\text { Wavelength } \\
\text { range }\end{array}$ & $\begin{array}{c}\text { Pixel field } \\
\text { of view }\end{array}$ & $\begin{array}{c}\text { Raster } \\
\text { size }\end{array}$ \\
\hline \multirow[t]{4}{*}{28 Apr. 1996} & 50201.45 & $10: 55-11: 03$ & $L W 3$ & $12-18 \mu \mathrm{m}$ & $6^{\prime \prime} \times 6^{\prime \prime}$ & $2 \times 2$ \\
\hline & & 11:03-11:10 & $L W 7$ & $8.5-10.7 \mu \mathrm{m}$ & $6^{\prime \prime} \times 6^{\prime \prime}$ & $2 \times 2$ \\
\hline & & $11: 10-11: 17$ & $L W 1$ & $4-5 \mu \mathrm{m}$ & $6^{\prime \prime} \times 6^{\prime \prime}$ & $2 \times 2$ \\
\hline & & $11: 17-11: 25$ & $L W 8$ & $10.7-12 \mu \mathrm{m}$ & $6^{\prime \prime} \times 6^{\prime \prime}$ & $2 \times 2$ \\
\hline \multirow[t]{2}{*}{28 Apr. 1996} & 50201.48 & $11: 26-11: 35$ & $L W 2$ & $5-8.5 \mu \mathrm{m}$ & $3^{\prime \prime} \times 3^{\prime \prime}$ & $2 \times 2$ \\
\hline & & & $J$ & $1.25 \pm 0.15 \mu \mathrm{m}$ & $0.125^{\prime \prime} \times 0.125^{\prime \prime}$ & $2 \times 2$ \\
\hline \multirow[t]{2}{*}{28 Apr. 1996} & 50201.47 & $11: 15-11: 27$ & $H$ & $1.65 \pm 0.15 \mu \mathrm{m}$ & $0.125^{\prime \prime} \times 0.125^{\prime \prime}$ & $2 \times 2$ \\
\hline & & & $K_{\mathrm{s}}$ & $2.15 \pm 0.15 \mu \mathrm{m}$ & $0.125^{\prime \prime} \times 0.125^{\prime \prime}$ & $2 \times 2$ \\
\hline 20 Oct. 1997 & 50741.94 & $22: 26-22: 42$ & $L W 10$ & $8-15 \mu \mathrm{m}$ & $1.5^{\prime \prime} \times 1.5^{\prime \prime}$ & $2 \times 2$ \\
\hline \multirow[t]{6}{*}{24 Oct. 1997} & 50745.68 & $16: 22-16: 41$ & $L W 3$ & $12-18 \mu \mathrm{m}$ & $3^{\prime \prime} \times 3^{\prime \prime}$ & $3 \times 3$ \\
\hline & & $16: 41-17: 00$ & $L W 7$ & $8.5-10.7 \mu \mathrm{m}$ & $3^{\prime \prime} \times 3^{\prime \prime}$ & $3 \times 3$ \\
\hline & & 17:00-17:19 & $L W 9$ & $14-16 \mu \mathrm{m}$ & $3^{\prime \prime} \times 3^{\prime \prime}$ & $3 \times 3$ \\
\hline & & $17: 19-17: 38$ & $L W 6$ & $7-8.5 \mu \mathrm{m}$ & $3^{\prime \prime} \times 3^{\prime \prime}$ & $3 \times 3$ \\
\hline & & $17: 38-17: 57$ & $L W 8$ & $10.7-12 \mu \mathrm{m}$ & $3^{\prime \prime} \times 3^{\prime \prime}$ & $3 \times 3$ \\
\hline & & $17: 57-18: 16$ & $L W 4$ & $5.5-6.5 \mu \mathrm{m}$ & $3^{\prime \prime} \times 3^{\prime \prime}$ & $3 \times 3$ \\
\hline 25 Oct. 1997 & 50746.02 & $00: 22-00: 47$ & $L W 2$ & $5-8.5 \mu \mathrm{m}$ & $1.5^{\prime \prime} \times 1.5^{\prime \prime}$ & $3 \times 3$ \\
\hline
\end{tabular}

${ }^{a}$ Modified Julian Date $=$ Julian Date -2400000.5 .

Table 2. Infrared flux densities of GRS $1915+105 . F_{\mathrm{obs}}$ is the observed flux directly measured on ISOCAM images, except for the $J, H$ and $K$ band fluxes which were reported from Mahoney et al. (1997) and transformed into mJy units. $F_{\text {der }}$ is the resulting flux after dereddening with two different laws: from Lutz et al. (1996) and from Draine (1989).

\begin{tabular}{|c|c|c|c|c|c|c|}
\hline \multirow[t]{2}{*}{$\overline{\text { Filter }}$} & \multicolumn{2}{|c|}{$\bar{F} F_{\text {obs }}(\mathrm{mJy})$} & \multicolumn{2}{|c|}{ 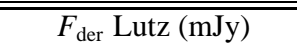 } & \multicolumn{2}{|c|}{ 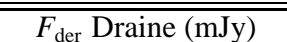 } \\
\hline & 1996 & 1997 & 1996 & 1997 & 1996 & 1997 \\
\hline$L W 1(4-5 \mu \mathrm{m})$ & $11.2 \pm 3.4$ & & $42.1 \pm 13$ & & $19.2 \pm 5.8$ & \\
\hline$L W 2(5-8.5 \mu \mathrm{m})$ & $5.39 \pm 1.6$ & $9.77 \pm 2.9$ & $17.4 \pm 5.2$ & $31.5 \pm 14$ & $7.72 \pm 2.3$ & $14.0 \pm 4.2$ \\
\hline$L W 4(5.5-6.5 \mu \mathrm{m})$ & & $11.6 \pm 3.5$ & & $37.1 \pm 11$ & & $16.0 \pm 4.8$ \\
\hline$L W 6(7-8.5 \mu \mathrm{m})$ & & $11.9 \pm 3.6$ & & $38.4 \pm 12$ & & $19.5 \pm 5.9$ \\
\hline$L W 7(8.5-10.7 \mu \mathrm{m})$ & $1.53 \pm 0.5$ & $4.87 \pm 1.5$ & $7.37 \pm 2.2$ & $23.5 \pm 7.0$ & $7.26 \pm 2.2$ & $23.1 \pm 6.9$ \\
\hline$L W 8(10.7-12 \mu \mathrm{m})$ & $3.54 \pm 1.1$ & $6.58 \pm 2.0$ & $10.3 \pm 3.1$ & $19.2 \pm 5.8$ & $10.8 \pm 3.2$ & $20.1 \pm 6.0$ \\
\hline$L W 9(14-16 \mu \mathrm{m})$ & & $13.8 \pm 4.1$ & & $23.9 \pm 7.2$ & & $24.0 \pm 7.2$ \\
\hline$L W 3(12-18 \mu \mathrm{m})$ & $5.34 \pm 1.6$ & $10.5 \pm 3.1$ & $9.25 \pm 2.8$ & $18.2 \pm 5.5$ & $9.29 \pm 2.8$ & $18.3 \pm 5.5$ \\
\hline$L W 10(8-15 \mu \mathrm{m})$ & & $14.7 \pm 4.4$ & & $41.3 \pm 12$ & & $36.0 \pm 11$ \\
\hline$J(1.25 \pm 0.15 \mu \mathrm{m})$ & $0.688 \pm 0.04$ & & $121.0 \pm 7.8$ & & $135.2 \pm 8.7$ & \\
\hline$H(1.65 \pm 0.15 \mu \mathrm{m})$ & $3.14 \pm 0.14$ & & $78.3 \pm 3.6$ & & $77.1 \pm 3.6$ & \\
\hline$K_{\mathrm{s}}(2.15 \pm 0.15 \mu \mathrm{m})$ & $6.98 \pm 0.26$ & & $52.7 \pm 1.9$ & & $51.8 \pm 1.9$ & \\
\hline
\end{tabular}

used an aperture correction to obtain the observed flux $F_{\text {obs }}$. The photometry accuracy was estimated to $\sim 30 \%$ mainly because of measurement errors due to the presence of this bright star.

As shown in Table 1, GRS 1915+105 was observed at different epochs with different filters and different spatial resolutions which raises doubt about whether the flux variability is real or not. Comparison with the bright source shows that its flux is stable on all our observations (within the error bars) and so the variability of the flux densities measured for GRS 1915+105 between 1996 and 1997 shown in Table 2 is real.

\subsection{Near-IR values}

We have looked for simultaneous observations of GRS 1915+105 with our ISOCAM ones and we have found that Mahoney et al. (1997) observed the source in the $J, H$ and $K_{\mathrm{s}}(2.15 \mu \mathrm{m})$ bands on April 281996 at the same time as ISOCAM $L W 1, L W 8$ and $L W 2$ filters as shown in Table 1. In this table the $J, H$ and $K_{\mathrm{s}}$ band fluxes were reported from Mahoney et al. (1997) after conversion from magnitude into milliJansky units according to Zombeck (1990) reference fluxes. They were dereddened using the Lutz et al. (1996) and Draine (1989) laws as described below in Sect. 3.2. These laws give at these 3 near-IR bands very similar results to the more commonly used Cardelli et al. (1989) law, so as they were used in the mid-IR range we kept them in the near-IR for coherence. Error bars on these bands were calculated by adding observational errors given by Mahoney et al. (1997) and errors caused by dereddening, about $10 \%$ for $J$ and $2 \%$ for $H$ and $K_{\mathrm{s}}$, so they are respectively $12 \%, 5 \%$ and $4 \%$.

We have also looked for the maximum and minimum published values in the $J, H$ and $K$ bands. The minima come from 
Chaty et al. (1996), the maxima come from Mahoney et al. (1997), Mirabel et al. (1996) and Chaty et al. (1996) for the $J, H$ and $K$-bands. We transformed these fluxes into milliJansky units and dereddened them.

\section{The dereddening problem}

\subsection{Distance and visible absorption}

The distance of GRS $1915+105$ is not known precisely. Observation of the superluminal ejecta enables us to constrain the maximum distance. Fender et al. (1999a) calculated their relativistic proper motion and found $11.2 \pm 0.8 \mathrm{kpc}$ as an upper limit. They also discussed the minimum distance, which is $>6.6 \mathrm{kpc}$. We adopt the value of $11.2 \mathrm{kpc}$.

At such a distance and because GRS $1915+105$ is located in the galactic plane with $\alpha=19^{\mathrm{h}} 15^{\mathrm{m}} 11.5494^{\mathrm{s}}$ and $\delta=$ $+10^{\circ} 56^{\prime} 44.758^{\prime \prime}$ in J2000 coordinates (Dhawan et al. 2000) corresponding to $l=45.3657^{\circ}$ and $b=-0.219^{\circ}$, the visible radiation from this source is heavily absorbed by the material along the line of sight. The optical extinction $A_{V}$ was roughly evaluated to be $A_{V} \sim 30 \mathrm{mag}$ by Mirabel et al. (1994) and $A_{V} \simeq 28$ mag by Boër et al. (1996). Chaty et al. (1996) derived from millimeter ${ }^{12} \mathrm{CO}(J=1-0)$ observations a total hydrogen column density $N_{\mathrm{H}}=4.7 \pm 0.2 \times 10^{22} \mathrm{~cm}^{-2}$ along the line of sight corresponding to $A_{V}=26.5 \pm 1 \mathrm{mag}$. This value was recently corrected by Chapuis \& Corbel (2003, in prep.) who find $N_{\mathrm{H}}=3.5 \pm 0.3 \times 10^{22} \mathrm{~cm}^{-2}$ and thus $A_{V}=19.6 \pm 1.7 \mathrm{mag}$. In this article we adopt $A_{V}=20 \mathrm{mag}$.

Note that this absorption coefficient is the main parameter used to deredden optical and infrared fluxes. With $A_{V}=20$ and using either the Rieke \& Lebofsky (1985) or the Cardelli et al. (1989) law one finds $A_{K}=2.2$ (see also Chapuis \& Corbel 2003 , in prep.) and not the 3.3 value, considered as a possible significant overestimate by Fender \& Pooley (1998) but widely used in previous articles with near-IR observations. Then for example the observed $\sim 3 \mathrm{mJy} K$-band oscillations amplitude leads when dereddened not to $\sim 60 \mathrm{mJy}$ but to $\sim 20 \mathrm{mJy}$ which is quite different from the radio oscillation amplitude. As a consequence the $K$-band flux in general is not as high as previously cited and thus the radio to IR spectrum is not as flat (Fender et al. 1997; Fender \& Pooley 2000), although the simultaneous radio and millimetre observations (Fender \& Pooley 2000) in which there are no dereddening uncertainties show a quite flat spectrum. However the flat spectrum argument may not be used against the van der Laan (1966) model of expanding clouds of plasma.

\subsection{Dereddening}

Mid-IR data need specific dereddening as the absorption law is highly wavelength dependent and irregular in this wavelength range compared to the near-IR range. The Cardelli et al. (1989) or Mathis (1990) laws are no longer valid for $\lambda>2.5 \mu \mathrm{m}$ (so in the mid-IR) as they totally ignore typical features such as the ones due to silicates. The galactic interstellar medium was studied separately by Draine (1989) and Lutz et al. (1996) who found two different absorption laws, particularly in the

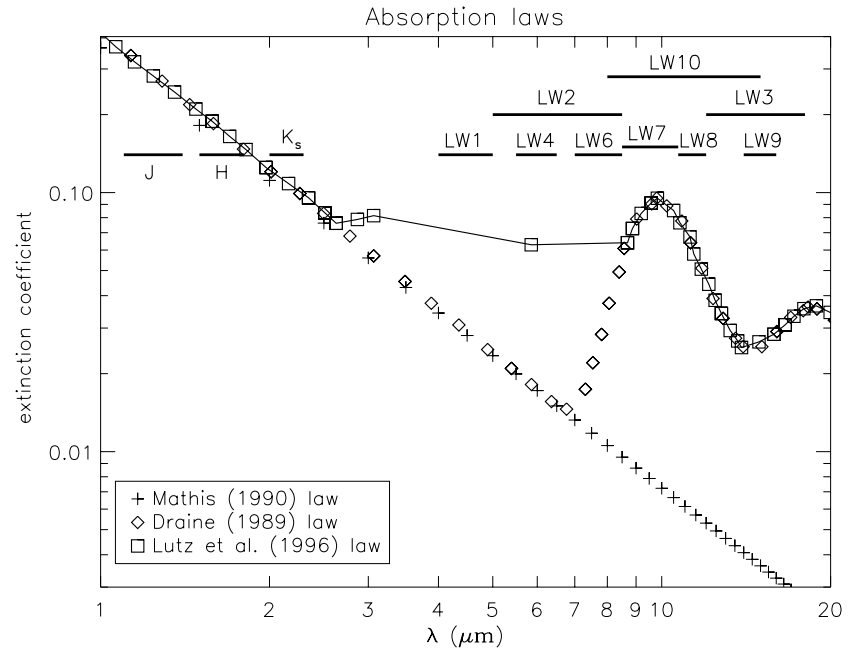

Fig. 1. Comparison of the near-IR absorption law by Mathis (1990) where $A_{\lambda} \propto \lambda^{-1.7}$ (plus sign) with Draine (1989) and Lutz et al. (1996) laws (respectively diamonds and squares) in the mid-IR. The extinction coefficient is defined by $A_{\lambda} / A_{V}$ where $A_{V}$ is the exctinction in the optical $V(0.55 \mu \mathrm{m})$ band and $A_{\lambda}$ is the exctinction at wavelength $\lambda$. The near-IR and ISOCAM filter wavelength ranges used in this article are overplotted.

$2.5-8.5 \mu \mathrm{m}\left(3.5-12 \times 10^{4} \mathrm{GHz}\right)$ range. Figure 1 shows the comparison between the Mathis (1990), Draine (1989) and Lutz et al. (1996) laws. According to Anthony Jones (priv. comm.) the Lutz et al. (1996) law applies for the Galactic center, for compact H II regions and for particular sources surrounded by high carbon density regions. The Draine (1989) law is supposed to apply in more diffuse regions so for the majority of Galactic sources. GRS $1915+105$ is not in the Galactic center region but it is in the dense Sagittarius arm, and we do not know its chemical environment. Thus, we could not choose between one or other law, so we have dereddened the flux densities with both laws and have studied each case in the following sections.

Note that what we call dereddened flux $F_{\text {der }}$ is linked to the observed flux $F_{\text {obs }}$ by the formula: $F_{\text {der }}=F_{\text {obs }} \times 10^{+0.4 A_{\lambda}}$ where $A_{\lambda}$ is the extinction at wavelength $\lambda$, calculated as $A_{V} \times$ the extinction coefficient at $\lambda$ given by either Draine (1989) or Lutz et al. (1996) law.

Dereddening introduces an error of about $5 \%$ into the flux density so it is negligible compared to the $30 \%$ error due to the photometry. Thus the total error on ISOCAM dereddened flux densities is $\sim 30 \%$ of the flux densities (see Table 2 ).

\section{A high/soft state in 1996?}

\subsection{State of the source}

We observed GRS $1915+105$ with ISOCAM for the first time on April 281996 (MJD 50201). Observed and dereddened fluxes are given in Table 2.

Because of the high variability of GRS $1915+105$, it is important to know the state of this source for data interpretation. The radio and X-ray monitorings of Fig. 2 illustrate the changes in the flux densities. The quick-look results provided by the ASM/RXTE team shows the integrated count rate of 

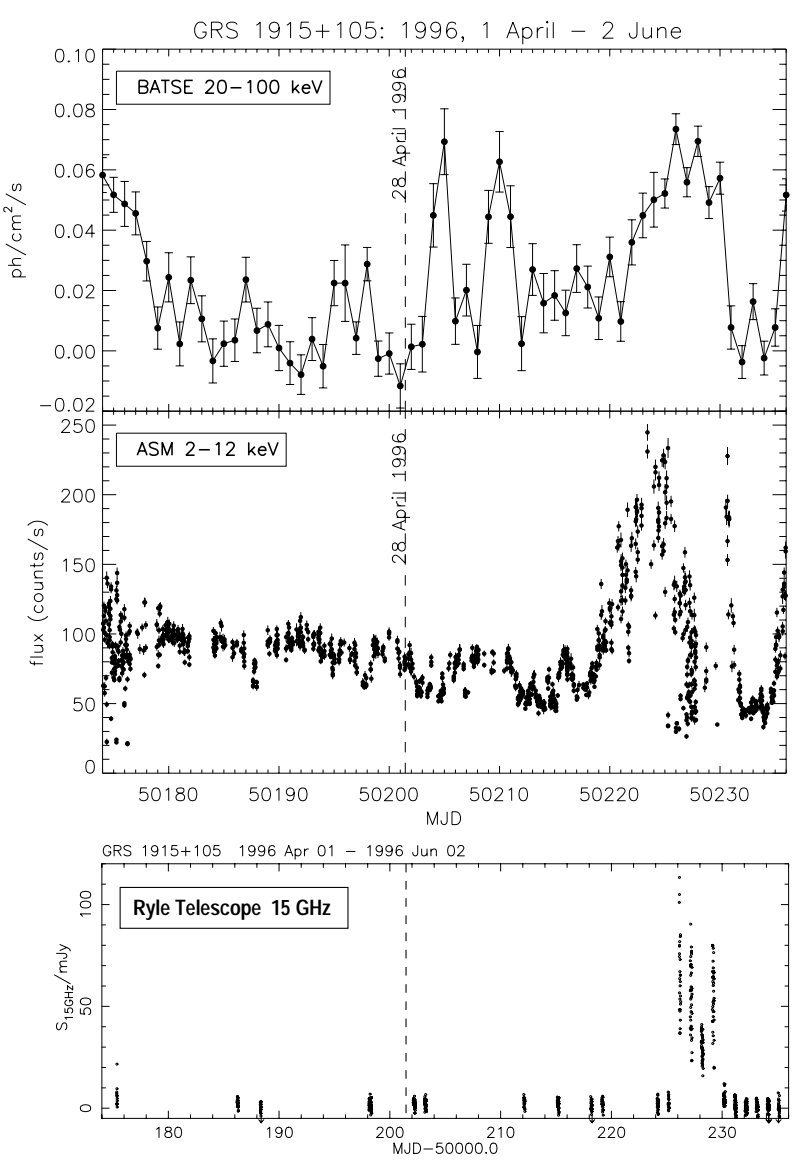

Fig. 2. X-ray and radio flux monitoring of GRS $1915+105$ around April 281996 (MJD 50201). The dotted line marks the begining of our ISOCAM observation on that day. The upper panels show the photon flux measured by BATSE in the $20-100 \mathrm{keV}$ band and the integrated count rate in the $2-12 \mathrm{keV}$ band by the Rossi X-ray Timing Explorer All-Sky Monitor (RXTE ASM). The lower panel shows the corresponding flux density measured at $15 \mathrm{GHz}$ with the Ryle Telescope (plot kindly provided by Guy Pooley).

GRS $1915+105$ over the $2-12 \mathrm{keV}$ band. The source behaviour was quite variable during the month preceeding and following our IR observations (see also the light curve in Fig. 4 of Pooley \& Fender 1997). On MJD 50201, GRS 1915+105 showed a relatively high soft X-ray flux around 80 counts/s. The corresponding BATSE occultation data showed no detectable hard $\mathrm{X}$-ray flux in the $20-100 \mathrm{keV}$ range on the same day. Thus, the state of GRS 1915+105 during our IR observations is moderately high and moderately soft.

There was no simultaneous radio observation of this period as the Green Bank Interferometer (GBI) was not operating. The Ryle Telescope (RT) was observing GRS1915+105 at $15 \mathrm{GHz}$ but not continuously as shown in Fig. 2 (RT plot kindly provided by Guy Pooley). There were observations taken on MJD 50199 and MJD 50202 i.e. about 2 days before and one day after our ISOCAM observation (see also Pooley $\&$ Fender 1997). They show a very low flux level of about $3 \pm 3 \mathrm{mJy}$ classified as a detection (Pooley priv. comm.) so the source was in a low radio state through the period of our observation.

\subsection{Results}

In order to understand the nature of the IR emission from GRS $1915+105$, we have tried to fit it using simple models. $\mathrm{X}$-ray binaries gather many sources of IR radiation: the donor star, the external edge of the accretion disc, thermal reprocessing of the X-ray disc flux, the basis of possible compact jets, heated dust around the system...

The most obvious IR source is the donor star. It was identified by Greiner et al. (2001b) as a K-M giant star with a temperature estimate of $\sim 4800_{-500}^{+200} \mathrm{~K}$ and a magnitude of $K=14.5-15.0$ uncorrected for extinction. Greiner et al. (2001a) estimated the Roche lobe size to be $21 \pm 4 R_{\odot}$. We have fitted the star contribution with a black body at a distance of $11.2 \mathrm{kpc}$, with a temperature $T$ and radius $R$. We have chosen the lowest temperature $T=4300 \mathrm{~K}$ since it corresponds to the most displaced spectral curve toward the mid-IR range. We have found the black body radius $R=19-23.5 R_{\odot}$ by comparing the resulting black body flux in the $K$-band with the dereddened $K$ magnitude. Finally we have chosen $R=23.5 R_{\odot}$ in order to model the star contribution with the highest flux density. This black body is plotted with the dashed-dotted line in Fig. 3 where it is clear that this contribution is faint in both near-IR and mid-IR range.

Thus other contributions are needed to explain the IR emission. Near-IR fluxes can be approximatively fitted by a power law $\left(F_{v} \propto v^{\alpha}\right)$ with a spectral index $\alpha=2$ (thin solid line in Fig. 3) but its interpretation is questionable. It may correspond to optically thick free-free emission but it would be observed at unusually high frequencies. This power law may also be due to the external edge of the accretion disc at a temperature of $\sim 8000 \mathrm{~K}$ (and radius $a \sim 35 R_{\odot}$ ), it would be the low energy tail of the multi-black body model commonly used to account for the majority of the soft X-ray emission in the high/soft state. However, as shown in Fig. 3 this power law emission is not sufficient to explain the mid-IR emission. Thus an additional component is needed in the mid-IR range. This component may be thermal emission from dust as plotted with the long-dashed line in Fig. 3 using a simple black body model with $T=400 \mathrm{~K}$ and $R=650 R_{\odot}$ (with Lutz et al. 1996) or $R=680 R_{\odot}$ (with Draine 1989).

The sum of the 3 components is plotted with a thick solid line in Fig. 3, but the result is moderately satisfactory for flux densities dereddened with the Draine (1989) law and not satisfactory for flux densities dereddened with the Lutz et al. (1996) law.

\subsection{Interpretation: A possible flaring event?}

This non-satisfactory fit of the April 281996 (MJD 50201) observations may be due to a flare occuring at the same moment in GRS $1915+105$.

Pooley \& Fender (1997) described MJD 50135-50220 as a period of varying degree of activity but with no significant radio emission apart from one small event. Their Fig. 4 shows indeed a quiet radio period but with a rather sparse coverage with the Ryle Telescope at $15 \mathrm{GHz}$. Nevertheless, it is possible that a brief flare or a brief and isolated flaring period had 

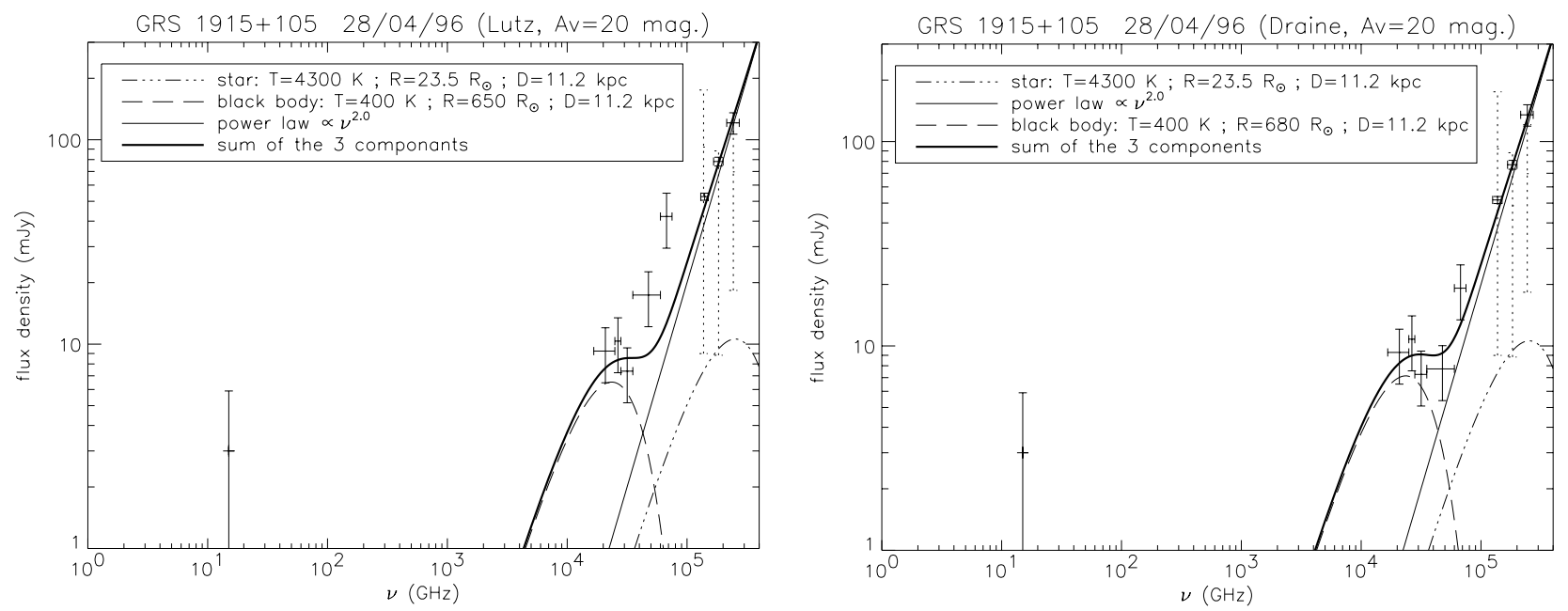

Fig. 3. IR spectrum of GRS $1915+105$ in 1996 April 28, dereddened with the (left) Lutz et al. (1996) and (right) Draine (1989) laws. The dotted lines correspond to the minima and maxima flux densities published in the $J\left(1.25 \mu \mathrm{m}=2.4 \times 10^{5} \mathrm{GHz}\right), H\left(1.65 \mu \mathrm{m}=1.81 \times 10^{5} \mathrm{GHz}\right)$ and $K\left(2.2 \mu \mathrm{m}=1.36 \times 10^{5} \mathrm{GHz}\right)$ bands. ISOCAM filters are, from left to right: $L W 3\left(1.67-2.5 \times 10^{4} \mathrm{GHz}\right), L W 8\left(2.5-2.8 \times 10^{4} \mathrm{GHz}\right)$, $L W 7\left(2.8-3.53 \times 10^{4} \mathrm{GHz}\right), L W 2\left(3.53-6.0 \times 10^{4} \mathrm{GHz}\right), L W 1\left(6.0-7.5 \times 10^{4} \mathrm{GHz}\right)$ (see Table 1). The low radio level observed with the Ryle Telescope at $15 \mathrm{GHz} 2$ days before and one day after the ISOCAM observations was added. Tentative fits of the IR flux densities are plotted as indicated in the legend (see details in the text).

occurred just during our ISOCAM observation since the $J$-band flux measured by Mahoney et al. (1997) is the highest ever reported for this wavelength filter and since very short flares can occur as the one seen just before MJD 50440 in Fig. 4 of Pooley \& Fender (1997). This possible brief IR flare could have occurred simultaneously in the radio wavelengths but as it lasted less than one day, no trace is left on the MJD 50202 Ryle Telescope observation.

This case assumes that the near-IR observations of Mahoney et al. (1997) must no longer be considered as simultaneous. Indeed it seems unlikely when one compares the near-IR observations which lasted 12 min with 4 min for each band (Mahoney et al. 1997) with the typical 30 min duration of the quasi-periodic oscillations of GRS 1915+105. For example, Fender et al. (1997) show oscillations with a flare duration of $\sim 15 \mathrm{~min}$, so 3 observations of 4 min each taken during this kind of oscillation can lead to very different flux levels and consequently to a big slope, as the one between the $J, H$ and $K_{\mathrm{s}}$ bands in Fig. 3. The extrapolation of this slope is not consistent with a detectable radio signal and this would be completely inconsistent with all the past recorded radio flares corresponding to high mean flux densities of the order of 25 to $50 \mathrm{mJy}$.

This possible flaring event is not identified as a peculiar variability of the flux evolution in each successive image taken with ISOCAM, perhaps because of the slow response of the mid-IR detectors emphasized by the weakness of the source. Thus flux densities measured in the different ISOCAM filters during this flare have to be considered as time averages (about 7 min per filter). As a consequence, the resulting spectral energy distribution does not represent a unique spectrum of GRS $1915+105$ and one should not rely too heavily on its modelisation. Near-IR oscillations observed as $\sim 30 \mathrm{~min}$ dip/flare cycles in the past were considered as synchrotron emission (see Sect. 1.1).
Another case to take into account is if no flare occurred during our ISOCAM observation on MJD 50201. Then one has to explain why the $J$-band flux observed by Mahoney et al. (1997) is the highest ever recorded. A possible explanation would be that the accretion disc had been particularly bright at that time, leading to high near-IR flux densities coming from the outer edge of the accretion disc, but it is unlikely as the RXTE/ASM flux was not exceptionally high.

Thus, the only reliable argument is that the donor star cannot account for most of the near and mid-IR emission of the source. One or several additional components are needed to explain this 1996 observation, such as synchrotron emission from a flaring event or thermal emission from the accretion disc external edge or from dust surrounding the binary system.

\section{The plateau state in 1997}

\subsection{State of the source}

The second set of ISOCAM observations of GRS 1915+105 took place in 1997 October as noted in Table 1. Figure 4 shows the X-ray and radio flux monitoring of the source during this month and the begining of 1997 November. Because of the quasi permanent and non predictable variability of the source in the IR, we have only plotted in Fig. 5 flux densities measured continuously on October 241997 (MJD 50745).

The GBI flux densities of GRS $1915+105$ and their estimated $1 \sigma$ errors on this day were at about $2 \mathrm{~h} 30$ (UT): $F_{2.25 \mathrm{GHz}}=44 \pm 4 \mathrm{mJy}, F_{8.3 \mathrm{GHz}}=56 \pm 6 \mathrm{mJy}$, spectral in$\operatorname{dex} \alpha=0.18 \pm 0.15\left(F_{v} \propto v^{\alpha}\right)$ and at about 19h (UT): $F_{2.25 \mathrm{GHz}}=44 \pm 4 \mathrm{mJy}, F_{8.3 \mathrm{GHz}}=40 \pm 6 \mathrm{mJy}, \alpha=-0.08 \pm 0.18$. This last observation occurred less than one hour after the end of our ISOCAM observation so we have used it for our spectral fits (see next section) although we have plotted the fluxes corresponding to both hours in Fig. 5. On that day, X-ray fluxes show 


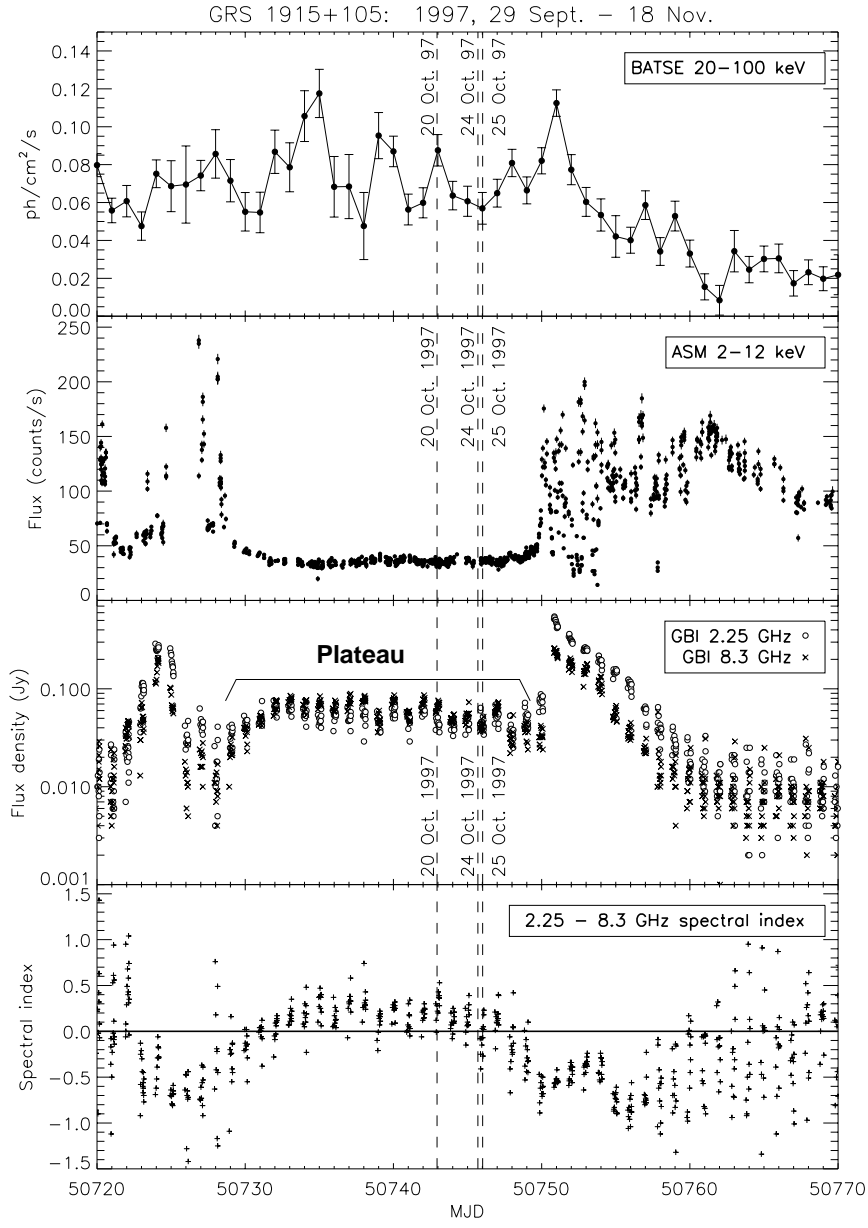

Fig. 4. X-ray and radio flux monitoring of GRS 1915+105 around October 241997 (MJD 50745). The upper panels show the photon flux measured by BATSE in the $20-100 \mathrm{keV}$ band and the integrated count rate in the $2-12 \mathrm{keV}$ band by the Rossi X-ray Timing Explorer All-Sky Monitor (RXTE ASM). The lower panels show the corresponding flux densities measured at $2.25 \mathrm{GHz}$ and $8.3 \mathrm{GHz}$ with the Green Bank Interferometer (GBI) and the spectral index from these two frequencies. On the latter plot the zero value has been marked with a solid line in order to emphasize the optically thick $(\alpha \sim 0)$ radio emission of the plateau state. The dotted lines mark the begining of our ISOCAM observations on 1997 October. They took place during this noticable plateau state preceding a giant radio outburst with superluminal ejections.

a low/hard state with a quite steady low RXTE/ASM 2-12 keV level of $\simeq 35$ counts/s and an irregular high BATSE $20-200 \mathrm{keV}$ photon flux of $\simeq 0.06 \mathrm{ph} / \mathrm{cm}^{2} / \mathrm{s}$.

At that epoch, the radio and X-ray monitorings show that GRS $1915+105$ was in a very particular state called the plateau state (see Figs. 4 and 1 of Fender et al. 1999a). This state, first described by Foster et al. (1996) and more specified by Fender et al. (1999a), results in an increase in the radio flux density to levels of $\sim 50-100 \mathrm{mJy}$ with an onset and a decay which can be as short as $\sim 1$ day. The plateau state is characterized by an optically thick radio emission $(\alpha \sim 0)$ and by a hard X-ray emission with a fairly high BATSE $20-100 \mathrm{keV}$ flux associated to a quasi-stable RXTE/ASM 2-12 keV flux. Its duration ranges from one day or two to many weeks. The inverted-spectrum radio emission $(\alpha \geq 0)$ corresponds to powerful self-absorbed quasi-continuous jets which were observed by Dhawan et al. (2000) and have spectral similarities with compact jets from other black hole candidate systems in similar hard X-ray states (Fender 2001). The plateau state also corresponds in $\mathrm{X}$-ray to the canonical low/hard $\mathrm{C}$ state class $\chi$ of Belloni et al. (2000a) dominated by a power-law component with little or no disc contribution in the X-ray band. More specifically this term associated with the presence of compact jets applies to $\chi_{1}$ and $\chi_{3}$ states as studied by Klein-Wolt et al. (2002), also called radio-loud or radio-plateau low/hard states by Muno et al. (2001) and type II hard steady states by Trudolyubov (2001). Vadawale et al. (2001) have even reported detection of synchrotron radiation in the $0.5-180 \mathrm{keV}$ X-ray band of RXTE PCA and HEXTE during this state, although it has to be confirmed.

Long (i.e. more than a few days) plateau states appear to precede major optically thin radio flares corresponding to superluminal radio ejection events (see also Fender et al. 2002). Plateau states are thus of further interest in order to understand the release of major superluminal outflows. As shown in Fig. 4, our ISOCAM observation on October 241997 were followed by a major flare 5 days later (on MJD 50750) with superluminal motion observed by Fender et al. (1999a) with MERLIN. As shown by the GBI spectral index, we observed GRS 1915+105 at the transition period from optically thick toward optically thin radio emission preparing the major outburst.

\subsection{Results}

The ISOCAM flux densities on October 241997 show quite different spectral energy distributions depending on the dereddening law used (Lutz et al. 1996 or Draine 1989). As for the 1996 observations, we have fitted the mid-IR emission with very simple models given the large error bars on the ISOCAM flux densities $(\sim 30 \%)$. In Fig. 5 we have plotted the contribution of the donor star with the same parameters: $T=4300 \mathrm{~K}$, $R=23.5 R_{\odot}$ (dashed-dotted line).

As the source was in a plateau low/hard state, we have fitted the radio emission with a power law representing the synchrotron radiation from the compact jets (solid thin line). But whether in this state the synchrotron component extends to the IR range, as has been asserted by Fender (2001), is questioned. This is why we have tried the three following cases: (1) no break in the synchrotron emission, (2) a break occuring close to the mid-IR range (respectively at $9000 \mathrm{GHz}$ and $31000 \mathrm{GHz}$ for the Lutz et al. 1996 and Draine 1989 laws) so that the synchrotron emission contributes to the mid-IR flux densities, and (3) a break occuring before the mid-IR range so that the synchrotron emission is negligible at those frequencies. The optically thick part of this synchrotron component has been modeled using the GBI flux densities measured only $1 \mathrm{~h}$ after ISOCAM thus with the corresponding spectral index $\alpha=-0.08$. The slope of this synchrotron emission after the break $(\alpha=-0.8)$ has been fitted according to the ISOCAM flux densities in the $L W 4, L W 6$ and $L W 7$ filters dereddened with the Draine (1989) law. 

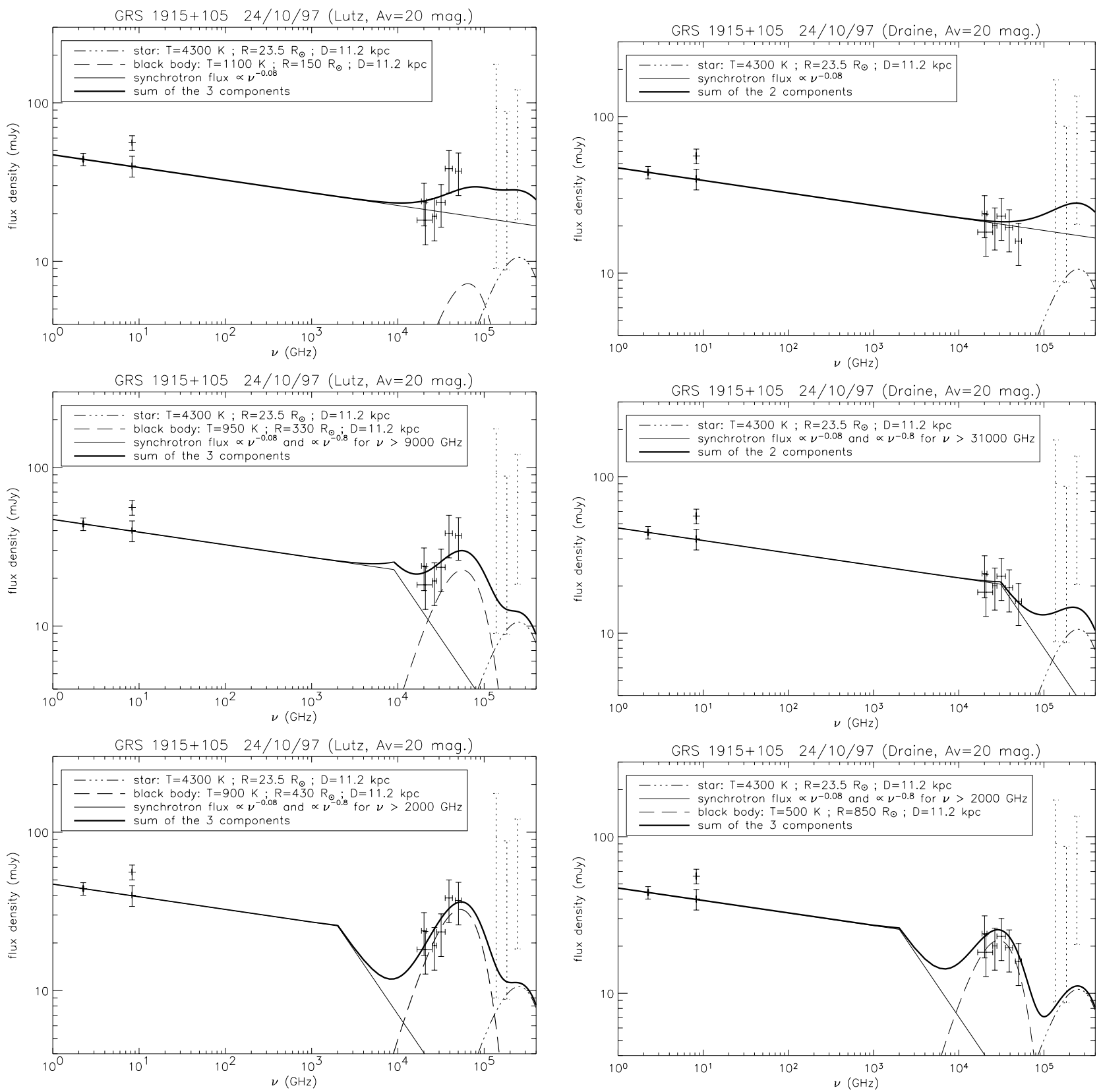

Fig. 5. Radio and ISOCAM spectrum of GRS 1915+105 in 1997 October 24, dereddened with (left) Lutz et al. (1996) and (right) Draine (1989) laws. The dotted lines correspond to the minima and maxima fluxes published in the $J\left(1.25 \mu \mathrm{m}=2.4 \times 10^{5} \mathrm{GHz}\right), H\left(1.65 \mu \mathrm{m}=1.81 \times 10^{5} \mathrm{GHz}\right)$ and $K\left(2.2 \mu \mathrm{m}=1.36 \times 10^{5} \mathrm{GHz}\right)$ bands. ISOCAM filters are, from left to right: $L W 3\left(1.67-2.5 \times 10^{4} \mathrm{GHz}\right), L W 9\left(1.875-2.14 \times 10^{4} \mathrm{GHz}\right)$, $L W 8\left(2.5-2.8 \times 10^{4} \mathrm{GHz}\right), L W 7\left(2.6-3.53 \times 10^{4} \mathrm{GHz}\right), L W 6\left(3.53-4.28 \times 10^{4} \mathrm{GHz}\right)$ and $L W 4\left(4.61-5.45 \times 10^{4} \mathrm{GHz}\right)(\mathrm{see}$ Table 1$)$. The GBI 2.25 and $8.3 \mathrm{GHz}$ fluxes observed $14 \mathrm{~h}$ before (higher level) and $1 \mathrm{~h}$ (lower level) after the ISOCAM observations are also plotted. These latter GBI values have been used in our tentative fits of the radio to IR fluxes which are plotted as indicated in the legend (see details in the text).

Figure 5 shows that case (1) can fit flux densities dereddened with the Draine (1989) law as they are grouped around the same level $(\sim 20 \mathrm{mJy})$ and the $\alpha=-0.08$ spectral index perfectly matches the mid-IR flux level despite the large error on this index $( \pm 0.18)$. But case (1) cannot fit flux densities dereddened with the Lutz et al. (1996) law where an additional component is clearly needed for the LW6 (3.53-4.28 × $\left.10^{4} \mathrm{GHz}=7-8.5 \mu \mathrm{m}\right)$ and $L W 4\left(4.61-5.45 \times 10^{4} \mathrm{GHz}=\right.$ 5.5-6.5 $\mu \mathrm{m}$ ) filters. Case (2) is satisfactory for flux densities dereddened with the Draine (1989) law if the break occurs in the mid-IR range $(31000 \mathrm{GHz}=9.7 \mu \mathrm{m})$. In all the other cases an additional component is needed. We then have used a black body model (long-dashed curves) standing for thermal emission from heated dust, with the following parameters: for the flux densities dereddened with the Lutz et al. (1996) law $T=1100 \mathrm{~K}$ and $R=150 R_{\odot}(1), T=950 \mathrm{~K}$ and $R=330 R_{\odot}$ (2), $T=900 \mathrm{~K}$ and $R=430 R_{\odot}$ (3); for the flux densities dereddened with the Draine (1989) law $T=500 \mathrm{~K}$ 
and $R=850 R_{\odot}$ in case (3). Note that these values must only be considered as orders of magnitude since the black body model is a very simple approximation for dust emission.

We can summarize the results of our fits, represented in Fig. 5 by the sums of the components in thick solid lines, as the following. The synchrotron emission extended to the midIR range can account for the flux densities in this wavelength range in the case of dereddening with the Draine (1989) law. In this latter case the mid-IR flux densities can also be explained by thermal emission from possible heated dust. Dereddening with the Lutz et al. (1996) law implies the need for a third component accounting for most of the mid-IR emission, possibly thermal emission from hot dust.

\subsection{Interpretation/discussion}

\subsubsection{Dust emission?}

If the mid-IR emission is due to hot dust, then one has to explain its presence and its heating mechanism.

First we question if the source of this dust could be the K-M giant donor star. Such a star has left the main sequence phase to evolve toward the AGB one. When it is isolated, its atmosphere expands and their mass loss increases to $\sim 10^{-9}-10^{-8} M_{\odot} \mathrm{yr}^{-1}$ during this giant phase. As GRS $1915+105$ is a binary system, the atmosphere expansion is limited by the Roche lobe size, and in this case the star has very likely reached its maximum size and has entered in the Roche lobe overflow phase with an accretion process characteristic of low-mass X-ray binaries. In order to produce dust, the material lost by the companion should escape from the black hole gravitational attraction, so the mass loss should exceed the mass accretion rate. This is unlikely as the latter has been evaluated by Belloni et al. (2000b) to $\sim 10^{-8}-10^{-7} M_{\odot} \mathrm{yr}^{-1}$ during IR quasi-periodic oscillations even if Belloni et al. (2000a) has estimated it to be very low during the plateau intervals. But even if the donor mass loss was high enough, the wind from a giant star is supposed to be composed of atomic elements and not of molecular ones which are on the other hand needed for dust production. Moreover the latter is usually not enabled with isolated giant stars since they are too hot. Thus it is unlikely that the donor star is a source of dust.

The dust could come from an external source of the binary system: the bright star seen in our ISOCAM images (see Sect. 2.1.2). This bright star is probably in the AGB phase according to the study of Felli et al. (2000). In order to stay consistent with this study, we took the magnitudes of this star from the ISOGAL survey (http://www-isogal.iap. fr/): $\simeq 7.99 \mathrm{mag}$ at $7 \mu \mathrm{m}(L W 2)$ and $\simeq 7.92 \mathrm{mag}$ at $15 \mu \mathrm{m}(L W 3)$ giving [7] $\simeq 7.43 \mathrm{mag}$ and [15] $\simeq 7.62 \mathrm{mag}$ when dereddened with $A_{V}=20 \mathrm{mag}$ and the relations $A_{7}=0.028 A_{V}$ and $A_{15}=0.015 A_{V}$ as in Felli et al. (2000), so [7]-[15] $\simeq 0.19 \mathrm{mag}$ which corresponds to an AGB class in Fig. 1 of Felli et al. (2000). If we assume that this star is located at the same distance from the Earth as GRS 1915+105, then the 18" angular separation between this AGB star and GRS 1915+105 corresponds to $\sim 0.58 \mathrm{pc}$ at $6.6 \mathrm{kpc}$ and $\sim 0.98 \mathrm{pc}$ at $11.2 \mathrm{kpc}$. An AGB star undergoes much mass loss (till $\sim 10^{-4} M_{\odot} \mathrm{yr}^{-1}$ ) and can eject material in its surroundings as far as $1 \mathrm{pc}$. But at such a distance only little material is provided and so it may not be able to account for the mid-IR emission from GRS 1915+105.

On the other hand, if we assume that this AGB star is much closer than GRS 1915+105, its surrounding nebula caused by the mass loss probably contaminates the line of sight to this source. This might explain the anomalous $\mathrm{Si}$ and $\mathrm{Fe}$ abundances deduced by Lee et al. (2002) from Chandra spectra of GRS $1915+105$, instead of dust that they suggested among other possibilities.

The problem is also to explain the high temperatures ( 900 to $1100 \mathrm{~K}$ ) of the dust and the distances found with our fits. The distance, 150 to $850 R_{\odot}$, represents 6 to 35 times the chosen radius of the giant star $\left(23.5 R_{\odot}\right)$ which is constrained by the gravitational interactions with the black hole ( $R \leq 21 \pm 4 R_{\odot}$ the Roche lobe size estimated by Greiner et al. 2001a). These parameters would not be surprising for dust surrounding some AGB stars, as shown by Danchi et al. (1994) who found M stars with inner dust shells very close to the photospheres of the stars (3-5 stellar radii) and at high temperature $(\sim 1200 \mathrm{~K})$. But for a giant star with a magnitude of $K=14.5-15.0$ as inferred by Greiner et al. (2001b) for GRS $1915+105$, the luminosity of the this donor star illustrated by the black body in dashed-dotted line in Fig. 5 is not high enough to induce the luminosity of the black body representing the possible dust on our fits. Then the dust heating in GRS $1915+105$ is unlikely to be carried out by the donor star.

Another possible origin of the dust heating might be the high energy activity of GRS $1915+105$. As a consequence, changes in the observed temperature of this dust between our fits in 1996 and in 1997 (when mid-IR flux densities is higher) might be due to changes in the X-ray activity. Thus the low/hard radiations coming from the jets or from Compton reprocessing of the soft disc photons by the corona would be more efficient than high/soft emission from the accretion disc. However, the efficiency of dust heating by X-rays is questioned by van Paradijs et al. (1994) who discussed the origin of the $10 \mu \mathrm{m}$ emission from the low-mass X-ray binary GRO J0422+32. They found, from the study of Voit (1991) on $\mathrm{X}$-ray irradiation of interstellar grains, that this mid-IR emission cannot be explained by the X-ray heating of dust, which leads to flux densities at least two orders of magnitude below the observed value, unless the system is surrounded by a highly non-standard interstellar medium.

Note that the hypothesis of IR emission due to dust surrounding GRS $1915+105$ was initially suggested by Mirabel et al. (1996) from the observation of an IR flare occuring 2 days after a radio flare and corresponding to a reddening between the $J$ and $K$ band observations taken quasi-simultaneously. However, at that epoch the $\sim 30$ min cycles of flares were not known and the observations in each near-IR band took about $10 \mathrm{~min}$. As a consequence these flux densities are time average ones and are not reliable for a spectral indication. It is very likely that on August 15 1995, the observed flare and reddening of GRS 1915+105 by Mirabel et al. (1996) were due to a 30 min flaring event since it happened 5 days after a giant radio outburst, now considered as the sign of superluminous ejections, and since Fender et al. (1999a, 2002) have observed 
these quasi-periodic oscillations in radio during the decay of such giant outbursts.

Thus if we draw the parallel with symbiotic stars (for a review see Mikolajewska 2003) GRS 1915+105 belongs to the $\sim 80 \%$ of S-type (stellar) systems containing a normal giant (the remaining $\sim 20 \%$ are D-type containing Mira-type variable with a dust shell). However it is a very peculiar one with a black hole instead of a white dwarf, with a short binary period and orbital separation leading to Roche lobe overflow instead of the high predominance of wind-accretion in usual symbiotic stars.

\subsubsection{Other: Synchrotron or free-free emission}

Thermal emission from dust seems so complicated to explain that another solution is more likely. As we observed GRS 1915+105 during the plateau state, which is known to correspond to the presence of a compact jet observed by Dhawan et al. (2000), the high-frequency extension of the radio synchrotron emission to the mid-IR logically ensues. This hypothesis was first suggested by Fender (2001) gathering the radio data of Pooley \& Fender (1997) with the only $K$-band flux from Bandyopadhyay et al. (1998) during the plateau state of 1996 August. However these observations were not simultaneous and Fig. 5 suggests that the $K$-band flux probably corresponds not only to synchrotron emission (if at all) but that the giant companion contribution may not be negligable in this band. In our study, using the Draine (1989) law, the extension of the radio synchrotron emission can account for the totality of the mid-IR emission measured in several bands. It may be the first time that we have evidence of synchrotron emission in mid-IR for GRS $1915+105$ in the plateau state. In this case, GRS $1915+105$ is similar to other black hole candidate systems in hard X-ray states showing powerful self-absorbed quasi-continuous jets (Fender 2001) with synchrotron emission extending to the near-IR or optical ranges such as GX 339-4 (Corbel \& Fender 2002), XTE J1118+480 (Markoff et al. 2001; Fender et al. 2001) and XTE J1550-564 (Corbel et al. 2001).

The synchrotron emission in the mid-IR range seems very likely but we cannot rule out another possibility corresponding to the flat spectrum obtained with the Draine (1989) law: optically thin free-free emission from an X-ray driven wind from the accretion disc. This kind of emission was suggested by van Paradijs et al. (1994) for GRO J0422+32 to explain their observed $10 \mu \mathrm{m}$ flux, although Fender (2001) suggests that this detection was also synchrotron emission from the compact selfabsorbed jet. The presence of a strong radiatively driven wind was also suggested by Lee et al. (2002) to explain ionized features in the X-ray spectrum of GRS $1915+105$, although evidence of this might be difficult to observe. Such a wind may be formed by X-ray heating of the disc for X-ray luminosities in excess of a few percent of the Eddington limit (Begelman et al. 1983) which is probably the case for GRS $1915+105$ (King 2002). Infrared free-free emission from a disc wind may be common for X-ray binaries as it has already been observed in Cygnus X-3 (Fender et al. 1999b; Koch-Miramond et al. 2002) and possibly in SS 433 (Wynn-Williams \& Becklin 1979; Fuchs et al. 2003, in prep.).
Note that either synchrotron or free-free emission corresponds to the flat spectrum resulting from dereddening with the Draine (1989) law. The latter appears more likely than the Lutz et al. (1996) law since it leads to satisfactory solutions to account for the mid-IR emission without the need for an additional component as thermal emission from dust (which is not easy to explain). Thus our 1997 observation enables us to prefer and to recommend the use of the Draine (1989) law to deredden the mid-IR observations of GRS 1915+105.

\section{Conclusions}

We have presented for the first time mid-IR observations of GRS 1915+105 obtained with ISOCAM. The near-IR and ISOCAM observations during the high/soft state in 1996 are difficult to interpret since they possibly took place during a flaring event with a too fast time variability compared to the response of the mid-IR detectors. The emission from the giant donor star alone cannot account for the near and mid-IR emission and one or several additional components are needed.

The other ISOCAM observation took place in October 1997, while GRS $1915+105$ was in the peculiar plateau state characterized by a flat or slightly inverted radio spectrum due to the synchrotron emission from compact jets. This state is of further interest as it preceded a giant radio flare corresponding to a major superluminal ejection event. We showed for the first time observations during a plateau state with quasi-simultaneous radio and mid-IR measurements. We discussed the different possibilities concerning the latter. Due to the large error-bars neither solution can be totally excluded but the presence of dust and its inferred temperature is unlikely.

Synchrotron emission can account for the flat broadband spectrum from the radio to the mid-IR range. Thus the midIR emission during the plateau state of GRS $1915+105$ is very likely to be the extension of the radio synchrotron emission, although optically thin free-free emission of an X-ray drivenwind from the accretion disc cannot be excluded. The 1997 observation enables us to choose between the two dereddening laws: the one from Draine (1989) giving a flatter spectrum in the mid-IR appears more plausible than the Lutz et al. (1996) law.

Acknowledgements. We warmly thank Anthony Jones, Vivek Dhawan, Claude Chapuis, Jerome Rodriguez and Tibault Le Bertre for very informative and useful discussions. We also thank Guy Pooley for kindly providing RT monitoring flux densities. The ISOCAM data presented in this paper were analysed using "CIA", a joint development by the ESA Astrophysics Division and the ISOCAM Consortium. The ISOCAM Consortium was led by the ISOCAM PI, C. Cesarsky, Direction des Sciences de la Matière, C.E.A, France. We thank the organisations providing the public data that we have used: quick-look results provided by the ASM/RXTE team, highlevel data products from the CGRO BATSE instrument which were generated by the BATSE Instrument Team at the Marshall Space Flight Center (MSFC) using the Earth occultation technique, and GBI monitoring programme. The Green Bank Interferometer is a facility of the National Science Foundation operated by the National Radio Astronomy Observatory, in support of USNO and NRL geodetic and astronomy programs, and of NASA High Energy Astrophysics programs. Y.F. is supported by a CNES external post-doctoral fellowship. 


\section{References}

Bandyopadhyay, R., Martini, P., Gerard, E., et al. 1998, MNRAS, 295, 623

Begelman, M. C., McKee, C. F., \& Shields, G. A. 1983, ApJ, 271, 70

Belloni, T., Mendez, M., King, A. R., van der Klis, M., \& van Paradijs, J. 1997a, ApJ, 488, L109

Belloni, T., Mendez, M., King, A. R., van der Klis, M., \& van Paradijs, J. 1997b, ApJ, 479, L145

Belloni, T., Klein-Wolt, M., Méndez, M., van der Klis, M., \& van Paradijs, J. 2000a, A\&A, 355, 271

Belloni, T., Migliari, S., \& Fender, R. P. 2000b, A\&A, 358, L29

Blandford, R. D., \& Konigl, A. 1979, ApJ, 232, 34

Boër, M., Greiner, J., \& Motch, C. 1996, A\&A, 305, 835

Cardelli, J. A., Clayton, G. C., \& Mathis, J. S. 1989, ApJ, 345, 245

Castro-Tirado, A. J., Brandt, S., \& Lund, N. 1992, IAU Circ., 5590

Castro-Tirado, A. J., Brandt, S., Lund, N., et al. 1994, ApJS, 92, 469

Cesarsky, C. J., Abergel, A., Agnese, P., et al. 1996, A\&A, 315, L32

Chapuis, C., \& Corbel, S. 2003, A\&A, submitted

Chaty, S., Mirabel, I. F., Duc, P. A., Wink, J. E., \& Rodríguez, L. F. 1996, A\&A, 310, 825

Corbel, S., \& Fender, R. P. 2002, ApJ, 573, L35

Corbel, S., Kaaret, P., Jain, R. K., et al. 2001, ApJ, 554, 43

Cotton, W. D., Counselman, C. C., Geller, R. B., et al. 1979, ApJ, 229, L115

Danchi, W. C., Bester, M., Degiacomi, C. G., Greenhill, L. J., \& Townes, C. H. 1994, AJ, 107, 1469

Delanay, M., \& ISOCAM Interactive Analysis Team. 2000, ESA Document SAI/96-5226/Dc, available at http://www.iso. vilspa.esa.es/archive/software/

Dhawan, V., Mirabel, I. F., \& Rodríguez, L. F. 2000, ApJ, 543, 373

Draine, B. T. 1989, in Infrared Spectroscopy in Astronomy, 93

Eikenberry, S. S., \& Fazio, G. G. 1997, ApJ, 475, L53

Eikenberry, S. S., Matthews, K., Morgan, E. H., Remillard, R. A., \& Nelson, R. W. 1998a, ApJ, 494, L61

Eikenberry, S. S., Matthews, K., Muno, M., et al. 2000, ApJ, 532, L33

Eikenberry, S. S., Matthews, K., Murphy., T. W., et al. 1998b, ApJ, 506, L31

Felli, M., Comoretto, G., Testi, L., Omont, A., \& Schuller, F. 2000, A\&A, 362, 199

Fender, R. P. 2001, MNRAS, 322, 31

Fender, R. P., Garrington, S. T., McKay, D. J., et al. 1999a, MNRAS, 304, 865

Fender, R. P., Hanson, M. M., \& Pooley, G. G. 1999b, MNRAS, 308, 473

Fender, R. P., Hjellming, R. M., Tilanus, R. P. J., et al. 2001, MNRAS, 322, L23

Fender, R. P., \& Pooley, G. G. 1998, MNRAS, 300, 573

Fender, R. P., \& Pooley, G. G. 2000, MNRAS, 318, L1

Fender, R. P., Pooley, G. G., Brocksopp, C., \& Newell, S. J. 1997, MNRAS, 290, L65

Fender, R. P., Rayner, D., Trushkin, S. A., et al. 2002, MNRAS, 330, 212

Foster, R. S., Waltman, E. B., Tavani, M., et al. 1996, ApJ, 467, L81

Fuchs, Y., Koch-Miramond, L., \& Ábrahám, P. 2003, A\&A, submitted
Greiner, J., Cuby, J. G., \& McCaughrean, M. J. 2001a, Nature, 414, 522

Greiner, J., Cuby, J. G., McCaughrean, M. J., Castro-Tirado, A. J., \& Mennickent, R. E. 2001b, A\&A, 373, L37

Greiner, J., Morgan, E. H., \& Remillard, R. A. 1996, ApJ, 473, L107

Harmon, B. A., Paciesas, W. S., \& Fishman, G. J. 1992, IAU Circ., 5619,2

Kaiser, C. R., Sunyaev, R., \& Spruit, H. C. 2000, A\&A, 356, 975

King, A. R. 2002, MNRAS, 335, L13

Klein-Wolt, M., Fender, R. P., Pooley, G. G., et al. 2002, MNRAS, 331,745

Koch-Miramond, L., Ábrahám, P., Fuchs, Y., Bonnet-Bidaud, J. M., \& Claret, A. 2002, A\&A, 396, 877

Lee, J. C., Reynolds, C. S., Remillard, R., et al. 2002, ApJ, 567, 1102

Lutz, D., Feuchtgruber, H., Genzel, R., et al. 1996, A\&A, 315, L269

Mahoney, W. A., Corbel, S., Durouchoux, P., et al. 1997, in Proceedings of the Fourth Compton Symposium, AIP Conf. Proc. 410, 912

Markoff, S., Falcke, H., \& Fender, R. 2001, A\&A, 372, L25

Martí, J., Mirabel, I. F., Chaty, S., \& Rodríguez, L. F. 2000, A\&A, 356, 943

Mathis, J. S. 1990, ARA\&A, 28, 37

Mikolajewska, J. 2003, Symbiotic stars probing stellar evolution, ASP Conf. Ser. [astro-ph/0210489]

Mirabel, I. F., Dhawan, V., Chaty, S., et al. 1998, A\&A, 330, L9

Mirabel, I. F., Duc, P. A., Rodríguez, L. F., et al. 1994, A\&A, 282, L17

Mirabel, I. F., Duc, P. A., Teyssier, R., et al. 1993a, IAU Circ., 5830, 1

Mirabel, I. F., \& Rodríguez, L. F. 1994, Nature, 371, 46

Mirabel, I. F., Rodríguez, L. F., Chaty, S., et al. 1996, ApJ, 472, L111

Mirabel, I. F., Rodríguez, L. F., Cordier, B., Paul, J., \& Lebrun, F. 1992, Nature, 358, 215

Mirabel, I. F., Rodríguez, L. F., Martí, J., et al. 1993b, IAU Circ., 5773, 2

Mirabel, I. F., \& Rodríguez, L. F. 1999, ARA\&A, 37, 409

Muno, M. P., Remillard, R. A., Morgan, E. H., et al. 2001, ApJ, 556, 515

Pooley, G. 1995, IAU Circ., 6269, 1

Pooley, G. 1996, IAU Circ., 6411, 1

Pooley, G. G., \& Fender, R. P. 1997, MNRAS, 292, 925

Rieke, G. H., \& Lebofsky, M. J. 1985, ApJ, 288, 618

Rodríguez, L. F., \& Mirabel, I. F. 1993, IAU Circ., 5900

Rodríguez, L. F., \& Mirabel, I. F. 1997, ApJ, 474, L123

Rodríguez, L. F., \& Mirabel, I. F. 1999, ApJ, 511, 398

Sams, B., Eckart, A., \& Sunyaev, R. 1996a, IAU Circ., 6455, 2

Sams, B. J., Eckart, A., \& Sunyaev, R. 1996b, Nature, 382, 47

Starck, J. L., Abergel, A., Aussel, H., et al. 1999, A\&AS, 134, 135

Trudolyubov, S. P. 2001, ApJ, 558, 276

Vadawale, S. V., Rao, A. R., \& Chakrabarti, S. K. 2001, A\&A, 372, 793

van der Laan, H. 1966, Nature, 211, 1131

van Paradijs, J., Telesco, C. M., Kouveliotou, C., \& Fishman, G. J. 1994, ApJ, 429, L19

Voit, G. M. 1991, ApJ, 379, 122

Wynn-Williams, C. G., \& Becklin, E. E. 1979, Nature, 282, 810

Zombeck, M. V. 1990, Handbook of space astronomy and astrophysics, 2nd ed. (Cambridge: University Press) 